\title{
Two-center convergent close-coupling calculations for positron-lithium collisions
}

\author{
A. V. Lugovskoy, ${ }^{*}$ A. S. Kadyrov, I. Bray, and A. T. Stelbovics \\ Department of Imaging and Applied Physics, \\ Curtin University, Perth 6150, Australia
}

(Dated: December 3, 2010)

\begin{abstract}
We report on two-center convergent close-coupling calculations of positron-lithium collisions. The target is treated as one active electron interacting with an inert ion core. The positronium formation channels are taken into account explicitly utilizing both negative- and positive-energy Laguerre-based states. A large number of channels and high partial waves are used to ensure the convergence of the cross sections. We find the Ramsauer-Townsend minimum in total and elastic cross sections at the impact energy $E$ about $0.0016 \mathrm{eV}$. As found previously for $\mathrm{H}$ and $\mathrm{He}$, the contribution to the break-up cross section from both the Li and Ps centers becomes the same as the threshold is approached.
\end{abstract}

PACS numbers: 34.85.1 x, 36.10.Dr, 34.70.1 e, 31.15.Ja

*Electronic address: A.Lugovskoy@curtin.edu.au 


\section{INTRODUCTION}

The physics of positron collisions with atomic targets is of practical and fundamental interest. Positron interactions with matter can be used to access a wealth of information on atomic and molecular structures and reaction mechanisms. Their understanding is crucial for development and improvement of a number of high-tech applications such as positronannihilation material analysis and cancer imaging. The two center nature of the problem, atom and positronium, generates particular challenge for theorists, while generation of suitable positron beams is a substantial experimental challenge.

The last decade has seen significant advent of low-energy trap-based positron beams [1-3]. New high-resolution experiments have been conducted for a number of atomic and molecular targets such as $\mathrm{He}$ [4], $\mathrm{Ne}$ and $\mathrm{Ar}$ [3], $\mathrm{CO}_{2}$ [5], $\mathrm{H}_{2}$ [6], $\mathrm{H}_{2} \mathrm{O}$ and $\mathrm{HCOOH}$ [7]. From the theoretical side there are several approaches to positron scattering such as, for example, the eikonal final state-continuum distorted wave approximation $[8,9]$, the exterior complex scaling method [10], the hybrid R-matrix approach [11], the momentum-space coupled-channel optical method [12] and the close-coupling method [13, 14].

In this paper we study positron scattering from atomic lithium. For positron collisions with alkalies both elastic and rearrangement channels are open at all incident energies. Hence, the valid theoretical description has to treat appropriately the "competition" between the positive-charge centers, $\mathrm{Li}^{+}$and positron, for the valence electron. However, the first attempt to treat the problem was to use the one-center expansion of the total wavefunction over atomic orbitals [15-17]. This counter-intuitive approach is consistent with the idea of basis completeness. But convergence of such expansions turned out to be very poor [18].

Another way to tackle the problem was to use the two-center expansion where both atom and positronium states are taken into account on equal footing. This approach resulted in better agreement with the experiment. For positron-lithium case the two-center expansion was employed by Guha and Ghosh [19], Basu and Ghosh [20], Abdel-Raouf [21], Hewitt et al. [22], McAlinden et al. [18] and Le et al. [23].

In this paper we report two-center convergent close-coupling (CCC) calculations of positron scattering by atomic lithium. Previously, this method was implemented for positron collisions with hydrogen [24] and helium [25, 26] targets. The usage of complete bases on both centers resolved the issue of unphysical resonances [24] and resulted in excellent agree- 
ment between the theory and experiment. Our purpose is to provide convergent results at energies where Ps formation is not negligible. At very low energies we identify a RamsauerTownsend minimum [19]. This structure not previously been found for this collision system. The paper is organized as follows. In Sec. II we describe the theoretical approach together with the used model potentials. The results are presented in Sec. III, followed by concluding remarks.

\section{THEORY}

In this paper we follow the approach where a positron-lithium collision is treated as a three-body problem. The interacting particles are the incoming positron, the active (outer shell) electron and the $\mathrm{Li}^{+}$ion. The 1 s electrons of the core do not participate directly in the collision event. They provide screening of the nucleus and take part in exchange with the active electron.

The scattering wave function $\Psi$ satisfies the Shrödinger equation

$$
(E-H) \Psi=0
$$

where $E$ is the total energy and

$$
H=H_{0}+V
$$

is the Hamiltonian of this system with $H_{0}$ and $V$ being, respectively, the three-free-particle Hamiltonian and the sum of all two-body interactions. The Hamiltonian $H_{0}$ is used in two different forms

$$
\begin{aligned}
H_{0} & =-(1 / 4) \nabla_{\rho_{\beta}}^{2}-\nabla_{r_{\beta}}^{2}, \\
& =-(1 / 2) \nabla_{\rho_{\alpha}}^{2}-(1 / 2) \nabla_{r_{\alpha}}^{2} .
\end{aligned}
$$

corresponding to two different sets of Jacobi coordinates $\left\{\boldsymbol{r}_{\alpha}, \boldsymbol{\rho}_{\alpha}\right\}$ and $\left\{\boldsymbol{r}_{\beta}, \boldsymbol{\rho}_{\beta}\right\}$, see Fig. 1. In this paper we follow the notations adopted in Ref. 27. In our case symbols $\alpha, e$ and $\beta$ point to individual particles - positron, electron and $\mathrm{Li}^{+}$ion, respectively. Also, they label the particle pairs so that $\alpha$ indicates the electron- $\mathrm{Li}^{+}, e$ - positron- $\mathrm{Li}^{+}$and $\beta$ - electron-positron pair. 


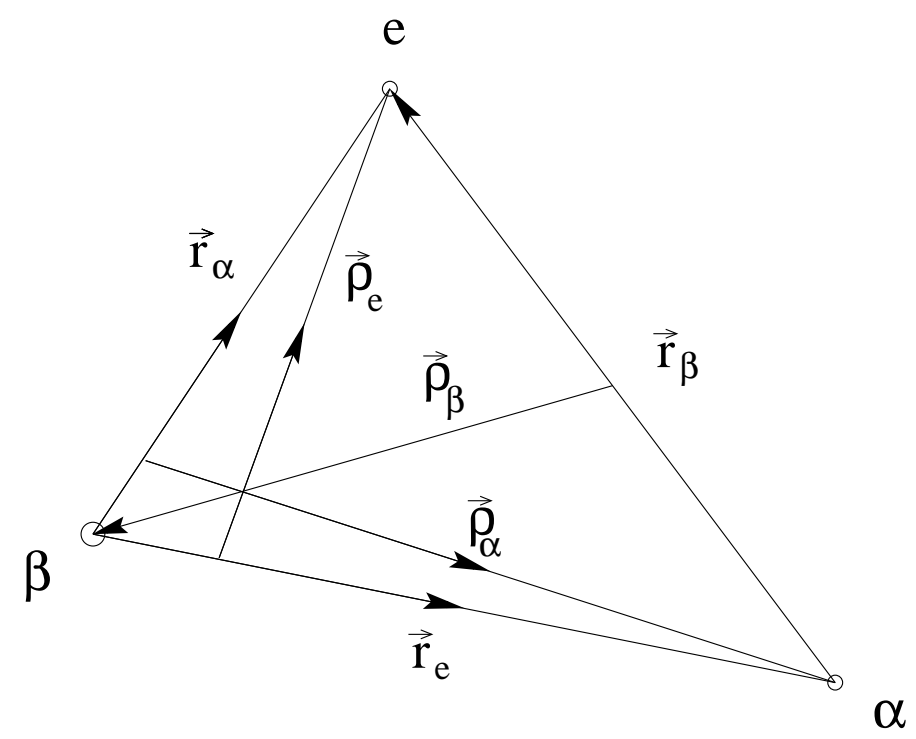

FIG. 1: Jacobi coordinates for positron $(\alpha)$, electron $(e)$ and $\mathrm{Li}^{+}$ion $(\beta)$.

For potential $V$ in Eq. (2) we use in pairs $\alpha, \beta$ and $e$

$$
V=V_{\alpha}\left(r_{\alpha}\right)+V_{e}\left(r_{e}\right)+V_{\beta}\left(r_{\beta}\right) .
$$

where the electron-ion and positron-ion terms, $V_{\alpha}$ and $V_{e}$, are

$$
\begin{aligned}
& V_{\alpha}(r)=V_{\mathrm{st}}(r)+V_{\mathrm{ex}}(r), \\
& V_{e}(r)=-V_{\mathrm{st}}(r),
\end{aligned}
$$

with $V_{\text {st }}$ and $V_{\text {ex }}$ being the static and exchange terms of the Hartree-Fock potential. The static term is calculated by

$$
V_{\text {st }}(r)=-\frac{Z}{r}+2 \sum_{\psi_{j} \in C} \int d^{3} r^{\prime} \frac{\left|\psi_{j}\left(\boldsymbol{r}^{\prime}\right)\right|^{2}}{\left|\boldsymbol{r}-\boldsymbol{r}^{\prime}\right|},
$$

where $Z$ is the charge of the nucleus and $\psi_{j}$ are the states of the ion core $C$ generated by performing the self-consistent-field Hartree-Fock calculations [28]. The summation in Eq. (8) is done for all core states. The exchange between the active electron and core electrons is taken into account in the framework of the equivalent local-exchange approximation [29-31]

$$
V_{\mathrm{ex}}\left(r, E_{\mathrm{ex}}\right)=\frac{1}{2}\left[\left(E_{\mathrm{ex}}-V_{\mathrm{st}}(r)\right)-\sqrt{\left(E_{\mathrm{ex}}-V_{\mathrm{st}}(r)\right)^{2}+\rho(r)}\right],
$$

where

$$
\rho(r)=\sum_{\psi_{j} \in C} \int \mathrm{d} \hat{\boldsymbol{r}}\left|\psi_{j}(\boldsymbol{r})\right|^{2}
$$


is the electron density distribution in the core and $E_{\text {ex }}$ is a some adjustment parameter. Finally, the electron-positron interaction $V_{\beta}$ in Eq. (5) is the Coulomb potential.

Following the two-center CCC approach [27], wavefunction $\Psi$ in Eq. (1) is sought as an expansion

$$
\Psi \approx \sum_{\alpha}^{N_{\alpha}} F_{\alpha}\left(\boldsymbol{\rho}_{\alpha}\right) \psi_{\alpha}^{N_{\alpha}}\left(\boldsymbol{r}_{\alpha}\right)+\sum_{\beta}^{N_{\beta}} F_{\beta}\left(\boldsymbol{\rho}_{\beta}\right) \psi_{\beta}^{N_{\beta}}\left(\boldsymbol{r}_{\beta}\right)
$$

where $\psi_{\alpha}^{N_{\alpha}}$ and $\psi_{\beta}^{N_{\beta}}$ are atomic and positronium pseudostates, respectively, and $F_{\alpha}$ and $F_{\beta}$ are their associated weight functions. The pseudostates, $\psi_{\alpha}^{N_{\alpha}}$ and $\psi_{\beta}^{N_{\beta}}$, are generated by diagonalizing the one-particle Hamiltonians [32]

$$
H_{\alpha}=-\frac{1}{2} \nabla_{\boldsymbol{r}}+V_{\alpha}(r)
$$

and

$$
H_{\beta}=-\frac{1}{4} \nabla_{\boldsymbol{r}}+V_{\beta}(r)
$$

using the square-integrable orthogonal Laguerre basis

$$
\xi_{n, l}(r)=\left(\frac{\lambda_{l}(n-1) !}{(2 l+1+n) !}\right)^{1 / 2}\left(\lambda_{l} r\right)^{l+1} \exp \left[-\lambda_{l} r\right] L_{n-1}^{2 l+2}\left(-\lambda_{l} r\right),
$$

where $L_{n-1}^{2 l+2}(x)$ is the associated Laguerre polynomial and $n$ ranges from 1 to the basis size $N_{l}$ for $l=0,1, \ldots, l_{\max }$. The complete sets of pseudostates contain both negative- and positive-energy states. Negative-energy states correspond to the bound states of the atomic target and positronium while positive-energy ones provide a discretization of their continuum spectra. The number of negative-energy states depends on parameters $\lambda_{l}$ and $N_{l}$ which are specific for every given orbital momentum number $l$.

Table I shows the energies of a few low levels resulted from the Hamiltonian dioganalization with $N_{l}=10-l$ and $N_{l}=50-l$, with $E_{\text {ex }}=-0.3831$ a.u.. These energy values are compared with the experimental data by Radziemski et al. [33] as well as the results of selfconsistent-field Hartree-Fock (SCFHF) calculations. The value of local-exchange parameter $E_{\text {ex }}$ was chosen so that the ground-level energy was equal to the experimental value. We see that the positions of the low-energy levels are well reproduced when the sufficiently big $N_{l}$ is used. For $N_{l}=50-l$ the largest relative error is $1.9 \%$. This is comparable with $1.2 \%$ error for the energy obtained with the SCFHF method. 
TABLE I: Experimental and theoretical energies of several low-lying levels of lithium in units of $\mathrm{eV}$.

\begin{tabular}{|c|c|c|c|c|}
\hline state & Exp & $\mathrm{SCHF}$ & $\mathrm{N}_{l}=10$ & $\mathrm{~N}_{l}=50$ \\
\hline $2 \mathrm{~s}$ & -5.392 & -5.342 & -5.392 & -5.392 \\
\hline $2 p$ & -3.544 & -3.501 & -3.614 & -3.614 \\
\hline $3 \mathrm{~s}$ & -2.019 & -2.009 & -2.009 & -2.019 \\
\hline $3 p$ & -1.558 & -1.544 & -1.540 & -1.579 \\
\hline $3 \mathrm{~d}$ & -1.513 & -1.512 & -1.493 & -1.514 \\
\hline $4 \mathrm{~s}$ & -1.051 & -1.047 & -0.589 & -1.051 \\
\hline $4 p$ & -0.870 & -0.865 & -0.144 & -0.879 \\
\hline $4 d$ & -0.851 & -0.850 & -0.316 & -0.851 \\
\hline
\end{tabular}

Substituting expansion (11) into (1) and following [27], one can derive the set of momentum-space coupled-channel equations for transition matrix elements

$$
T_{\gamma^{\prime}, \gamma}\left(\boldsymbol{q}_{\gamma^{\prime}}, \boldsymbol{q}_{\gamma}\right)=V_{\gamma^{\prime}, \gamma}\left(\boldsymbol{q}_{\gamma^{\prime}}, \boldsymbol{q}_{\gamma}\right)+\sum_{\gamma^{\prime \prime}}^{N_{\alpha}+N_{\beta}} \int \frac{d \boldsymbol{q}_{\gamma^{\prime \prime}}}{(2 \pi)^{3}} \frac{V_{\gamma^{\prime}, \gamma}\left(\boldsymbol{q}_{\gamma}^{\prime}, \boldsymbol{q}_{\gamma^{\prime \prime}}\right) T_{\gamma^{\prime}, \gamma}\left(\boldsymbol{q}_{\gamma^{\prime \prime}}, \boldsymbol{q}_{\gamma}\right)}{\left[E-\epsilon_{\gamma^{\prime \prime}}-q_{\gamma^{\prime \prime}}^{2} /\left(2 M_{\gamma^{\prime \prime}}\right)\right]},
$$

where $\boldsymbol{q}_{\gamma}$ is the momentum of the free particle $\gamma$ relative to the c.m. of the bound pair in channel $\gamma(\gamma=\alpha$ or $\beta), \epsilon_{\gamma}$ is the corresponding pseudoenergy of the bound pair, $M_{\gamma}$ is its reduced mass. The effective potential $V_{\gamma^{\prime}, \gamma}$ is defined as

$$
V_{\gamma^{\prime}, \gamma}\left(\boldsymbol{q}_{\gamma^{\prime}}, \boldsymbol{q}_{\gamma}\right)=\left\langle\boldsymbol{q}_{\gamma^{\prime}}\left|\left\langle\psi_{\gamma^{\prime}}\left|U_{\gamma^{\prime}, \gamma}\right| \psi_{\gamma}\right\rangle\right| \boldsymbol{q}_{\gamma}\right\rangle
$$

where $\left|\boldsymbol{q}_{\gamma}\right\rangle$ is a plane wave representing the free particle $\gamma$ and $U_{\gamma^{\prime}, \gamma}$ stands for one of the following channel operators

$$
U_{\alpha, \alpha}=V-V_{\alpha}, \quad U_{\beta, \beta}=V-V_{\beta}, \quad U_{\alpha, \beta}=H_{0}+V-E .
$$

By performing partial-wave expansion in the total orbital angular momentum $J$ one can get from Eq. (15)

$$
T_{\gamma^{\prime}, \gamma}^{L^{\prime} L J}\left(q_{\gamma^{\prime}}, q_{\gamma}\right)=V_{\gamma^{\prime}, \gamma}^{L^{\prime} L J}\left(q_{\gamma^{\prime}}, q_{\gamma}\right)+\sum_{\gamma^{\prime \prime}}^{N_{\alpha}+N_{\beta}} \sum_{L^{\prime \prime}} \int \frac{d q_{\gamma^{\prime \prime}} q_{\gamma^{\prime \prime}}^{2}}{(2 \pi)^{3}} \frac{V_{\gamma^{\prime}, \gamma}^{L^{\prime} L^{\prime \prime} J}\left(q_{\gamma}^{\prime}, q_{\gamma^{\prime \prime}}\right) T_{\gamma^{\prime}, \gamma}^{L^{\prime \prime} L J}\left(q_{\gamma^{\prime \prime}}, q_{\gamma}\right)}{\left[E+i 0-\epsilon_{\gamma^{\prime \prime}}-q_{\gamma^{\prime \prime}}^{2} /\left(2 M_{\gamma^{\prime \prime}}\right)\right]}
$$


where $V_{\gamma^{\prime}, \gamma}\left(\boldsymbol{q}_{\gamma^{\prime}}, \boldsymbol{q}_{\gamma}\right)$ and $V_{\gamma^{\prime}, \gamma}^{L^{\prime} L J}\left(q_{\gamma^{\prime}}, q_{\gamma}\right)$ (and similarly $T_{\gamma^{\prime}, \gamma}\left(\boldsymbol{q}_{\gamma^{\prime}}, \boldsymbol{q}_{\gamma}\right)$ and $T_{\gamma^{\prime}, \gamma}^{L^{\prime} L J}\left(q_{\gamma^{\prime}}, q_{\gamma}\right)$ ) are related to each other by

$$
V_{\gamma^{\prime}, \gamma}\left(\boldsymbol{q}_{\gamma^{\prime}}, \boldsymbol{q}_{\gamma}\right)=\sum_{L^{\prime}, M^{\prime}, L, M, J, K} Y_{L^{\prime} M^{\prime}}\left(\hat{\boldsymbol{q}}_{\gamma^{\prime}}\right) C_{L^{\prime} M^{\prime} l^{\prime} m^{\prime}}^{J K} V_{\gamma^{\prime}, \gamma}^{L^{\prime} L J}\left(q_{\gamma^{\prime}}, q_{\gamma}\right) C_{L M l m}^{J K} Y_{L M}^{*}\left(\hat{\boldsymbol{q}}_{\gamma}\right)
$$

and

$$
\begin{aligned}
V_{\gamma^{\prime}, \gamma}^{L^{\prime} L J}\left(q_{\gamma^{\prime}}, q_{\gamma}\right)= & \sum_{m^{\prime}, m, M^{\prime}, M} \iint \mathrm{d} \hat{\boldsymbol{q}}_{\gamma^{\prime}} \mathrm{d} \hat{\boldsymbol{q}}_{\gamma^{\prime}} \\
& \times Y_{L^{\prime} M^{\prime}}^{*}\left(\hat{\boldsymbol{q}}_{\gamma^{\prime}}\right) C_{L^{\prime} M^{\prime} l^{\prime} m^{\prime}}^{J K} V_{\gamma^{\prime}, \gamma}\left(\boldsymbol{q}_{\gamma^{\prime}}, \boldsymbol{q}_{\gamma}\right) C_{L M l m}^{J K} Y_{L M}\left(\hat{\boldsymbol{q}}_{\gamma}\right),
\end{aligned}
$$

where $Y_{L M}$ is a spherical harmonic and $C_{L^{\prime} M^{\prime} l^{\prime} m^{\prime}}^{J K}$ is a Clebsch-Gordan coefficient. The effective potentials $V_{\gamma^{\prime}, \gamma}^{L^{\prime} L J}\left(q_{\gamma^{\prime}}, q_{\gamma}\right)$ can be computed similarly to how it was done for the positron-hydrogen problem [27].

\section{MATRIX ELEMENTS}

Calculation of the effective potentials $V_{\gamma^{\prime}, \gamma}^{L^{\prime} L J}\left(q_{\gamma^{\prime}}, q_{\gamma}\right)$ is straightforward but tedious. In contrast to the hydrogen case there is no analytical expressions for the potentials $V_{e}(r)$ and $V_{\alpha}(r)$. These potentials are available in numerical form only. This means that we cannot use some intermediate analytical steps in calculation of the matrix elements which increases significantly the computation time.

To calculate matrix elements for direct (atom-atom and positronium-positronium) transitions one needs the spherical harmonic expansion of $V_{\alpha^{\prime}, \alpha}\left(\boldsymbol{q}_{\alpha^{\prime}}, \boldsymbol{q}_{\gamma}\right)$ and $V_{\beta^{\prime}, \beta}\left(\boldsymbol{q}_{\beta^{\prime}}, \boldsymbol{q}_{\beta}\right)$. In turn, this requires the spherical wave expansion of $U_{\alpha, \alpha}$ and $U_{\beta, \beta}$. Using the approximation $r_{e} \approx \rho_{\alpha}$ (atomic c.m. assumed to be at the ion center) one can get

$$
\begin{aligned}
U_{\alpha, \alpha} & =V-V_{\alpha}=V_{e}\left(r_{e}\right)+V_{\beta}\left(r_{\beta}\right) \\
& \approx \sum_{\lambda=0}^{\infty} \frac{4 \pi}{2 \lambda+1} U_{\alpha, \alpha}^{\lambda}\left(\rho_{\alpha}, r_{\alpha}\right)\left(\boldsymbol{Y}_{\lambda}\left(\hat{\rho}_{\alpha}\right) \cdot \boldsymbol{Y}_{\lambda}\left(\hat{r}_{\alpha}\right)\right),
\end{aligned}
$$

where

$$
U_{\alpha, \alpha}^{\lambda}\left(\rho_{\alpha}, r_{\alpha}\right)=V_{e}\left(\rho_{\alpha}\right) \delta_{\lambda, 0}-\frac{\min \left[r_{\alpha}, \rho_{\alpha}\right]^{\lambda}}{\max \left[r_{\alpha}, \rho_{\alpha}\right]^{\lambda+1}},
$$

and

$$
\left(\boldsymbol{Y}_{\lambda}\left(\hat{\rho}_{\alpha}\right) \cdot \boldsymbol{Y}_{\lambda}\left(\hat{r}_{\alpha}\right)\right)=\sum_{\mu=-\lambda}^{\lambda}\left(Y_{\lambda, \mu}^{*}\left(\hat{\rho}_{\alpha}\right) Y_{\lambda, \mu}\left(\hat{r}_{\alpha}\right)\right)
$$


Taking into account that $\boldsymbol{r}_{e}=-\boldsymbol{\rho}_{\beta}-\boldsymbol{r}_{\beta} / 2$ and $\boldsymbol{r}_{\alpha}=-\boldsymbol{\rho}_{\beta}+\boldsymbol{r}_{\beta} / 2$ we derive

$$
\begin{aligned}
U_{\beta, \beta} & =V-V_{\beta}=V_{e}\left(r_{e}\right)+V_{\alpha}\left(r_{\alpha}\right) \\
& =\sum_{\lambda} \frac{4 \pi}{2 \lambda+1} U_{\beta, \beta}^{(\lambda)}\left(\rho_{\beta}, r_{\beta}\right)\left(\boldsymbol{Y}_{\lambda}\left(\hat{\boldsymbol{\rho}_{\beta}}\right) \cdot \boldsymbol{Y}_{\lambda}\left(\hat{\boldsymbol{r}_{\beta}}\right)\right),
\end{aligned}
$$

where

$$
\begin{aligned}
U_{\beta, \beta}^{(\lambda)}\left(\rho_{\beta}, r_{\beta}\right)= & \frac{2 \lambda+1}{2} \int_{-1}^{1} d z P_{\lambda}(z)\left[V_{e}\left(\boldsymbol{\rho}_{\beta}+\frac{\boldsymbol{r}_{\beta}}{2}\right)+V_{\alpha}\left(\boldsymbol{\rho}_{\beta}-\frac{\boldsymbol{r}_{\beta}}{2}\right)\right] \\
= & \frac{2 \lambda+1}{2} \int_{-1}^{1} d z P_{\lambda}(z)\left\{V_{\alpha}\left(\sqrt{r_{<}^{2}+r_{>}^{2}-2 z r_{<} r_{>}}\right)\right. \\
& \left.+(-1)^{\lambda} V_{e}\left(\sqrt{r_{<}^{2}+r_{>}^{2}-2 z r_{<} r_{>}}\right)\right\}
\end{aligned}
$$

and where $P_{\lambda}(z)$ is the Legendre polynomial of degree $l, r_{<}=\min (\rho, r / 2)$ and $r_{>}=$ $\max (\rho, r / 2)$.

The matrix elements $V_{\beta, \alpha}\left(\boldsymbol{q}_{\beta}, \boldsymbol{q}_{\alpha}\right)$ for rearrangement transitions are presented as a sum of two terms, $V_{\beta, \alpha}^{(1)}\left(\boldsymbol{q}_{\beta}, \boldsymbol{q}_{\alpha}\right)$ and $V_{\beta, \alpha}^{(2)}\left(\boldsymbol{q}_{\beta}, \boldsymbol{q}_{\alpha}\right)$, calculated separately. The first term is

$$
\begin{aligned}
V_{\beta, \alpha}^{(1)}\left(\boldsymbol{q}_{\beta}, \boldsymbol{q}_{\alpha}\right) & =\iint d \boldsymbol{\rho}_{\beta} d \boldsymbol{r}_{\beta} e^{-i \boldsymbol{q}_{\beta} \cdot \boldsymbol{\rho}_{\beta}} \psi_{\beta}^{*}\left(\boldsymbol{r}_{\beta}\right)\left[\left(H_{0}-E\right)+V_{\beta}\left(r_{\beta}\right)+V_{\alpha}\left(r_{\alpha}\right)\right] \psi_{\alpha}\left(\boldsymbol{r}_{\alpha}\right) e^{i \boldsymbol{q}_{\alpha} \cdot \boldsymbol{\rho}_{\alpha}} \\
& =(2 \pi)^{3}\left[\mathcal{E}\left(\boldsymbol{q}_{\beta}, \boldsymbol{q}_{\alpha}\right) \tilde{\psi}_{\beta}^{*}\left(\boldsymbol{p}_{\beta}\right) \tilde{\psi}_{\alpha}\left(\boldsymbol{p}_{\alpha}\right)+\tilde{\psi}_{\beta}^{*}\left(\boldsymbol{p}_{\beta}\right) \tilde{g}_{\alpha}\left(\boldsymbol{p}_{\alpha}\right)+\tilde{g}_{\beta}^{*}\left(\boldsymbol{p}_{\beta}\right) \tilde{\psi}_{\alpha}\left(\boldsymbol{p}_{\alpha}\right)\right],
\end{aligned}
$$

where $\boldsymbol{p}_{\alpha}=\boldsymbol{q}_{\beta}-\boldsymbol{q}_{\alpha}, \boldsymbol{p}_{\beta}=\boldsymbol{q}_{\beta} / 2-\boldsymbol{q}_{\alpha}$, and $\mathcal{E}\left(\boldsymbol{q}_{\beta}, \boldsymbol{q}_{\alpha}\right)=p_{\alpha}^{2} / 2+q_{\alpha}^{2} / 2-E$. Functions with a tilde, $\tilde{\psi}_{i}(\boldsymbol{p})$ and $\tilde{g}_{i}(\boldsymbol{p})$, are the Fourier images of $\psi_{i}(\boldsymbol{r})$ and $V_{i}(r) \psi_{i}(\boldsymbol{r})$ respectively.

The second term $V_{\beta, \alpha}^{(2)}\left(\boldsymbol{q}_{\beta}, \boldsymbol{q}_{\alpha}\right)$ is

$$
\begin{aligned}
V_{\beta, \alpha}^{(2)}\left(\boldsymbol{q}_{\beta}, \boldsymbol{q}_{\alpha}\right) & =\iint d \boldsymbol{\rho}_{\beta} d \boldsymbol{r}_{\beta} e^{-i \boldsymbol{q}_{\beta} \cdot \boldsymbol{\rho}_{\beta}} \psi_{\beta}^{*}\left(\boldsymbol{r}_{\beta}\right) V_{e}\left(r_{e}\right) \psi_{\alpha}\left(\boldsymbol{r}_{\alpha}\right) e^{i \boldsymbol{q}_{\alpha} \cdot \boldsymbol{\rho}_{\alpha}} \\
& =(2 \pi)^{3} \int \frac{d \boldsymbol{p}}{(2 \pi)^{3 / 2}} \tilde{\psi}_{\beta}^{*}\left(\boldsymbol{p}_{\beta}^{\prime}\right) \tilde{V}_{e}\left(\boldsymbol{p}-\boldsymbol{q}_{\alpha}\right) \tilde{\psi}_{\alpha}\left(\boldsymbol{p}_{\alpha}^{\prime}\right),
\end{aligned}
$$

where $\boldsymbol{p}_{\beta}^{\prime}=\left(\boldsymbol{q}_{\beta} / 2\right)-\boldsymbol{p}, \boldsymbol{p}_{\alpha}^{\prime}=\boldsymbol{q}_{\beta}-\boldsymbol{p}$ and $\tilde{V}_{e}(\boldsymbol{p})$ is the Fourier transform of $V_{e}(\boldsymbol{r})$. Eq. (27) leads for the following expression for its reduced matrix element

$$
\begin{aligned}
\mathcal{V}_{\beta \alpha}^{L^{\prime} L(2)}\left(q_{\beta}, q_{\alpha}\right) & =\frac{1}{8 \pi^{3}} \int_{0}^{\infty} d q q^{2} Q_{L}^{\prime}\left(q, q_{\alpha}\right) \sum_{m, m^{\prime}, M, M^{\prime}} C_{M^{\prime} m^{\prime} K}^{L^{\prime}{ }^{\prime} J} C_{M m K}^{L l J} \\
& \times \iint d \hat{\boldsymbol{q}}_{\beta} d \hat{\boldsymbol{q}} Y_{L^{\prime} M^{\prime}}^{*}\left(\hat{\boldsymbol{q}}_{\beta}\right) Y_{L M}(\hat{\boldsymbol{q}}) \tilde{\psi}_{\beta}^{*}\left(\boldsymbol{p}_{\beta}^{\prime}\right) \tilde{\psi}_{\alpha}^{*}\left(\boldsymbol{p}_{\alpha}^{\prime}\right),
\end{aligned}
$$


where

$$
Q_{l}^{(v)}\left(q, q_{\alpha}\right)=2 \pi \int_{-1}^{1} P_{l}(z) V_{e}\left(\boldsymbol{q}-\boldsymbol{q}_{\alpha}\right) d z
$$

where $z=\cos (\alpha)$ and $\alpha$ is the angle between vectors $\boldsymbol{q}$ and $\boldsymbol{q}_{\alpha}$.

Calculation of matrix elements, especially those for rearrangement transitions, is the most time consuming part of the CCC calculations. The system of close-coupled equations we need to solve is ill-conditioned due to the usage of the two center expansion, requiring the matrix elements.

\section{RESULTS}

To obtain the transition matrix elements $T_{\gamma^{\prime}, \gamma}\left(\boldsymbol{q}_{\gamma^{\prime}}, \boldsymbol{q}_{\gamma}\right)$ the system of coupled momentumspace integral equations (17) is converted into equations for the $K$-matrix and then solved numerically using real arithmetic [32]. Calculations are performed for a limited number of partial waves $J$. We found that first ten partial waves are enough for get reliable results for the positronium formation cross sections at all energies. Direct scattering channels require at least ten partial waves more at the higher energies. Using the developed code we perform calculations with as many pseudostates from both centers as required for the convergence.

We conduct calculations with different basis sets to be sure that our results are independent of the set parameters such as exponential fall-off parameter $\lambda_{l}$, and convergent when increasing the parameters contributing to the basis size

$$
N=\sum_{l=0}^{l_{\max }^{\mathrm{Li}}} N_{l}^{\mathrm{Li}}+\sum_{l=0}^{l_{\max }^{\mathrm{Ps}}} N_{l}^{\mathrm{Ps}} .
$$

To make convergence issues simpler we set $\lambda_{l}^{\mathrm{Ps}}=\lambda^{\mathrm{Ps}}, \lambda_{l}^{\mathrm{Li}}=\lambda^{\mathrm{Li}}, l_{\max }^{\mathrm{Ps}}=l_{\max }^{\mathrm{Li}}=l_{\max }$ and $N_{l}^{\mathrm{Ps}}=N_{l}^{\mathrm{Li}}=N_{0}-l$. This way we reduce the number of parameters to just $N_{0}, l_{\max }, \lambda^{\mathrm{Li}}$ and $\lambda^{\mathrm{Ps}}$.

Given the commensurate treatment of both centers we need to demonstrate that convergence is possible, and that there is no double-counting of the ionization processes due to positive-energy states of both centers. We illustrate this in Fig. 2, within the S-wave model, where only zero orbital angular momenta are retained. Presented are the total break-up 


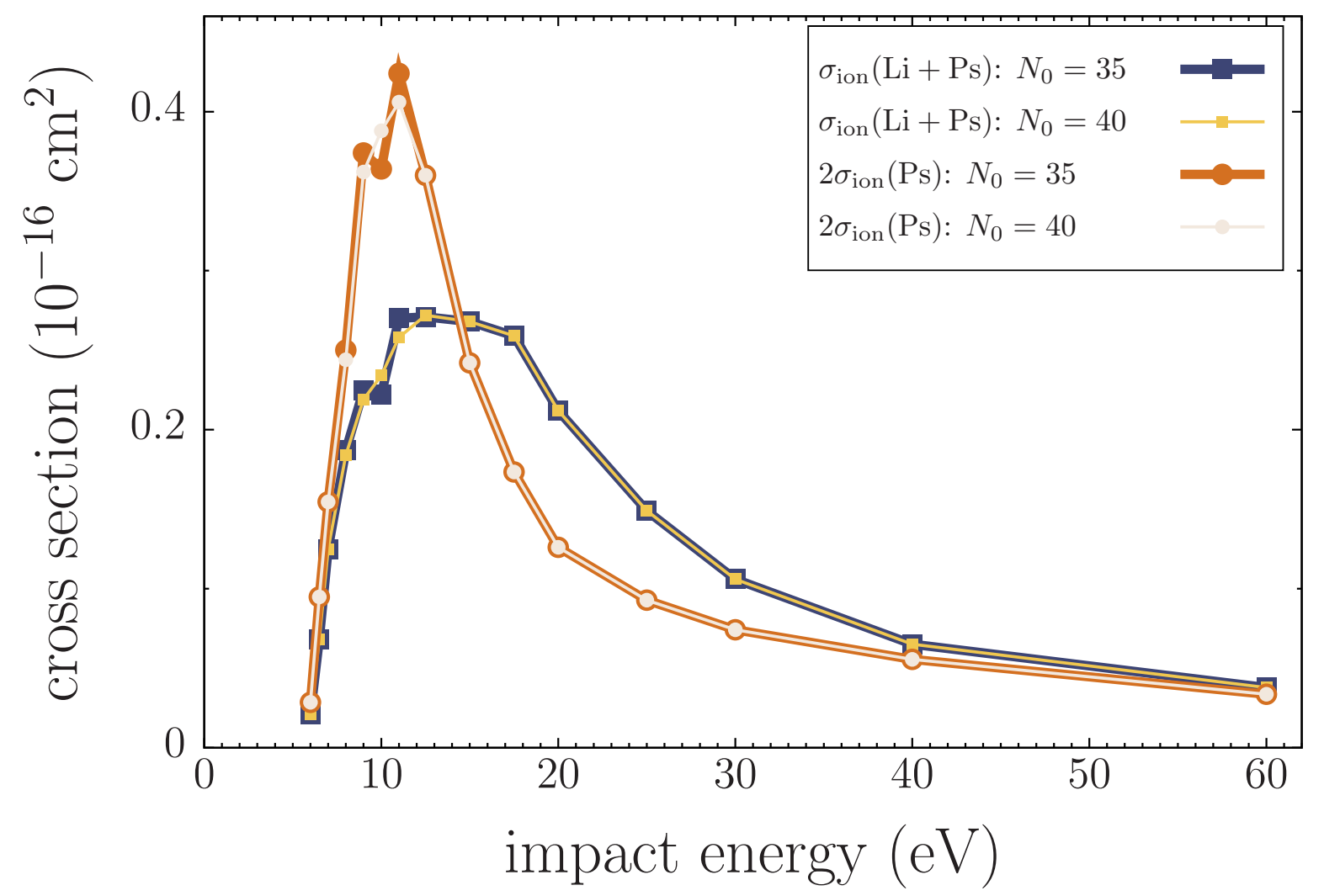

FIG. 2: Break-Up cross section $\sigma_{\text {ion }}(\mathrm{Li}+\mathrm{Ps})$ and $2 \sigma_{\text {ion }}(\mathrm{Ps})$ as functions of energy computed within the S-wave model, see text. The number of states used in calculations are indicated in the legend. cross section $\sigma_{\text {ion }}(\mathrm{Li}+\mathrm{Ps})$ as well as the positronium break-up cross section $\sigma_{\text {ion }}(\mathrm{Ps})$ multiplied by two. The first one equals to the sum of all cross sections over the positive-energy pseudostates of both centers

$$
\begin{aligned}
\sigma_{\text {ion }}(\mathrm{Li}+\mathrm{Ps}) & =\sum_{n: \epsilon_{n}^{\mathrm{Li}}>0} \sigma_{n}+\sum_{n: \epsilon_{n}^{\mathrm{Ps}}>0} \sigma_{n} \\
& \equiv \sigma_{\text {ion }}(\mathrm{Li})+\sigma_{\text {ion }}(\mathrm{Ps})
\end{aligned}
$$

while the second, $\sigma_{\text {ion }}(\mathrm{Ps})$, is due to the contributions of just the positronium positiveenergy pseudostates. We see excellent convergence for both $\sigma_{\text {ion }}(\mathrm{Li}+\mathrm{Ps})$ and $\sigma_{\text {ion }}(\mathrm{Ps})$ when the number of states on each center goes from 35 to 40. The two values of $N_{0}$ yield indistinguishable results at all energies except in the small region around $10 \mathrm{eV}$. Thus, the two independent $\mathrm{Li}$ and Ps contributions to the break-up cross section are independently convergent. Furthermore, the fact that the curves converge to each other at threshold indicates that there $\sigma_{\text {ion }}(\mathrm{Ps}) \approx \sigma_{\text {ion }}(\mathrm{Li})$. Previously, such behavior was demonstrated for the cases 

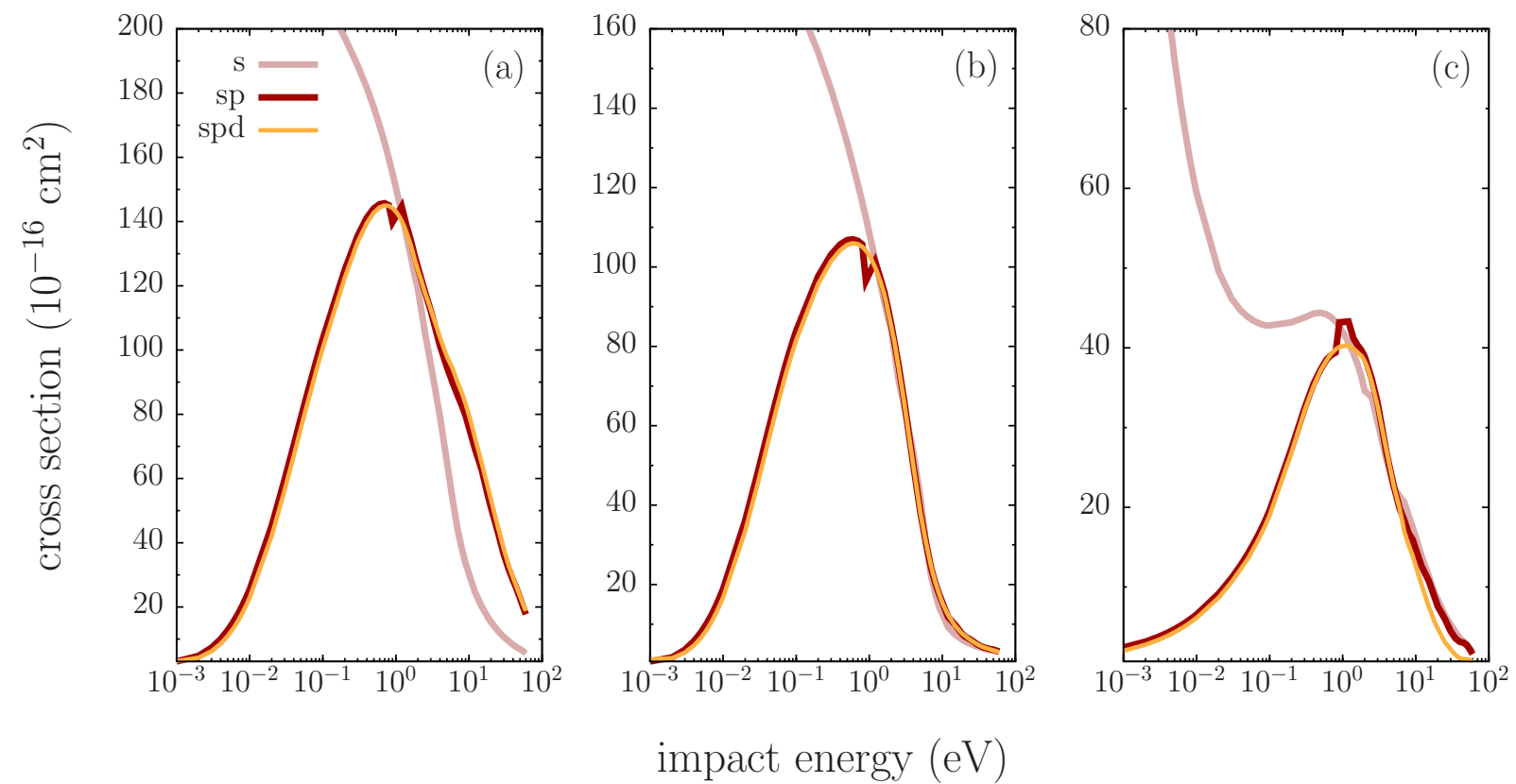

FIG. 3: (a) Total, (b) elastic and (c) positronium formation cross sections for positron-lithium collision calculated with the use of two-center CCC method. The number of basis states were the same for both centers and equal to $N_{0}=20$ s-states for the s-basis, $N_{0}=10$ and $N_{1}=9$ for the sp-basis, and $N_{0}=10, N_{1}=9$ and $N_{2}=8$ for the spd-basis.

of hydrogen [34] and helium [25]. We note that the system of equations becomes rapidly ill-conditioned as $N_{0}$ is increased.

Having established convergence in a model problem, we now consider the full problem. Figure 3 shows the total, elastic, and positronium formation cross sections as functions of the impact energy. They where calculated with the use of three different sets with $l_{\max }=0$ ( $\mathrm{s}$ basis), $l_{\max }=1$ (sp basis) and $l_{\max }=2$ (spd basis). Equal number of pseudostates were taken for both centers in our calculations. Figures 3 (a), (b) and (c) reveal the significant difference in the energy dependence between the results calculated with the s and sp bases. The differences between the sp- and spd-basis calculations are only marginal. These results suggest good convergence with $N_{0}$ and $l_{\max }$ for the presented transitions.

The grand total and elastic cross sections are presented in figures 4 and 5. They were calculated with the use of $N_{l}=10-l$ pseudostates for each center with $l_{\max }=2$. Also shown with black lines are calculations by McAlinden et al. [18]. We see that the results are generally in good agreement. The only difference is the resonant-like structure in the total 


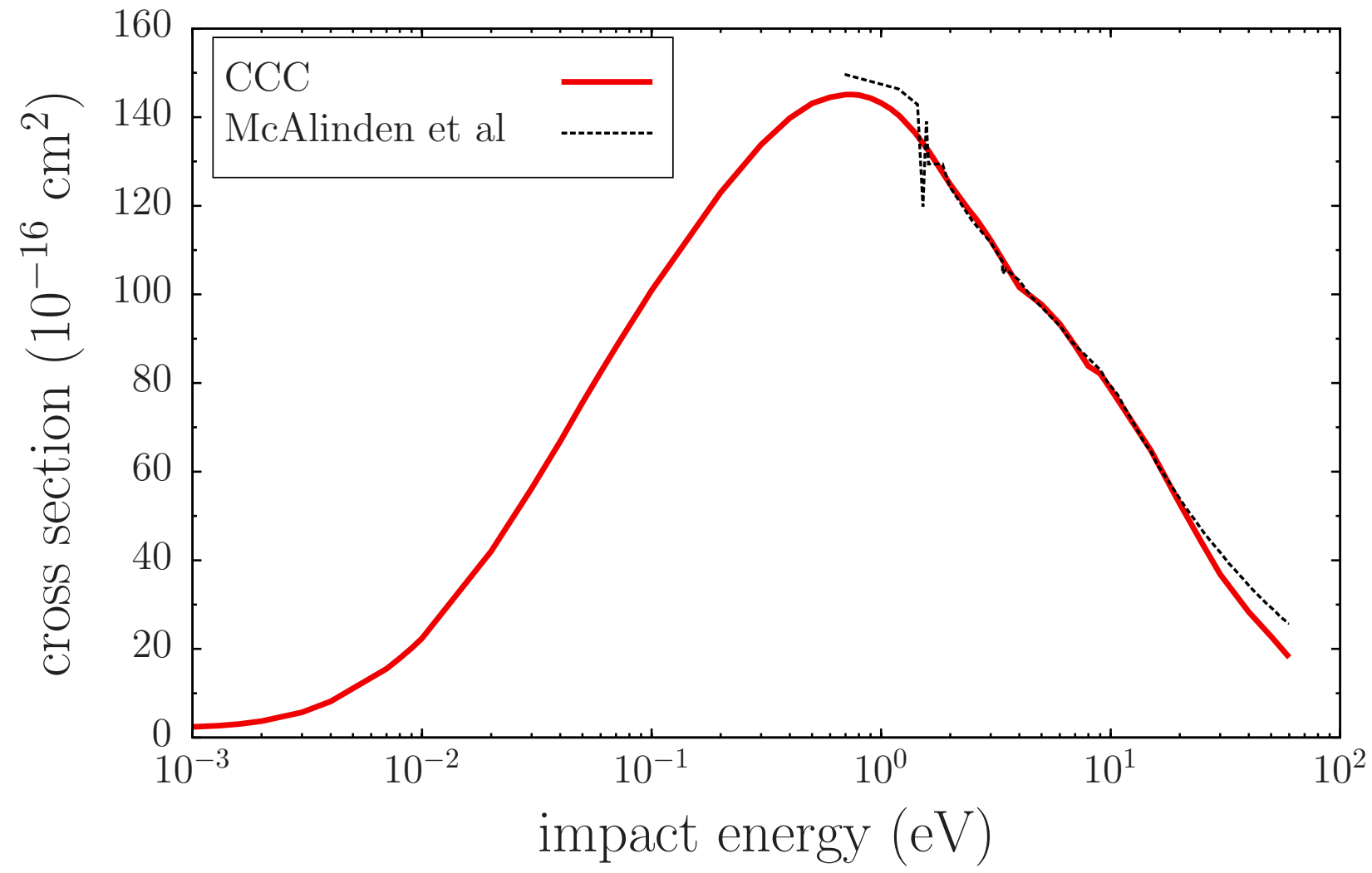

FIG. 4: Total cross sections for positron-lithium scattering. The CCC results (red lines) are compared with the data by McAlinden et al. [18] (black line). The spd-basis as in Fig. 3 was used in calculations.

cross section near $1.6 \mathrm{eV}$. We found this structure and its position to be basis-dependent. It disappears for sufficiently large bases on both centers. Also, for very low energies CCC predicts a shallow Ramsauer-like minimum in both total and elastic cross sections near $E=0.0016 \mathrm{eV}$ (see Fig. 5).

Figure 6 shows the positronium formation cross section. We compare our calculations (red solid and blue broken lines) with the experimental data by Surdutovich et al. [35] and theoretical results by McAlinden et al. [18] and Le et al. [23]. We also present results from a truncated basis that has only three eigenstates (1s, $2 \mathrm{~s}$ and $2 \mathrm{p}$ ) for positronium and twenty nine states (2s-9s, 2p-9p, 3d-9d and 4f-9f) for lithium. The states were chosen so that their energies were in close correspondence to the energies of the mixed-basis states used by McAlinden et al. [18].

We see that all theoretical curves are in overall qualitative agreement with each other. Our 


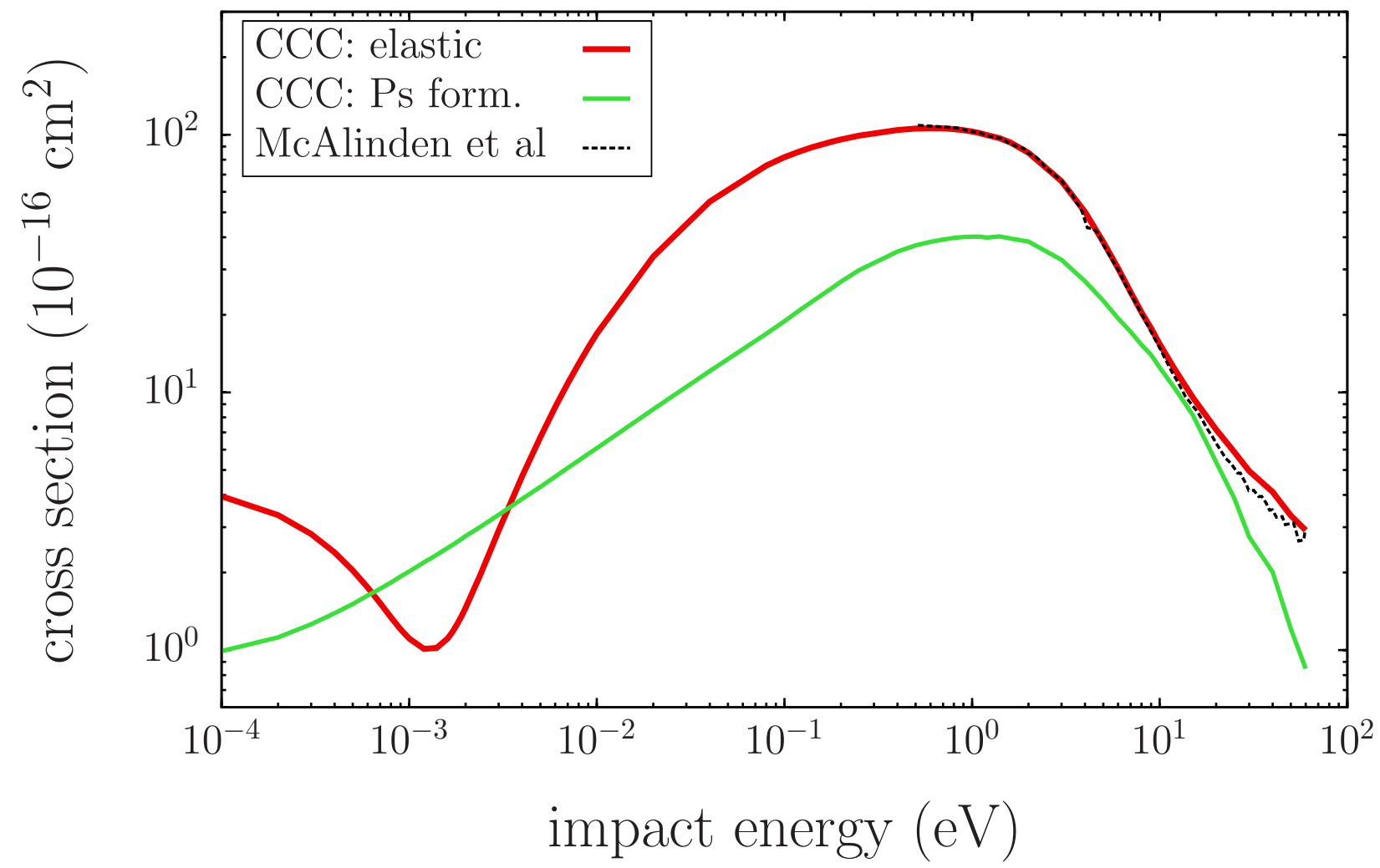

FIG. 5: Same as Fig. 4 but for the elastic cross sections. Also shown with the broken blue line is the positronium formation cross section. The CCC basis is the same as for Fig. 4.

truncated-basis calculations agree well with the results of McAlinden et al. [18], with both having a pseudoresonance, though at different energies. The differences can be attributed to the fact that we take into account the exchange part of the electron-electron interaction, and also use slightly different lithium states. The exchange interaction was also taken into account in the hyper-spherical close-coupling calculations [23]. The authors obtained a resonance-free energy dependence for the positronium formation cross section. We see that the only slight disagreement of the CCC and their results is in the magnitude of the peak. Agreement with experiment is not at the level that we would hope for this relatively simple collision system.

\section{CONCLUSION}

The two-center CCC method has been developed to calculate positron scattering with lithium atoms, where the positronium formation channel is taken into account explicitly. 


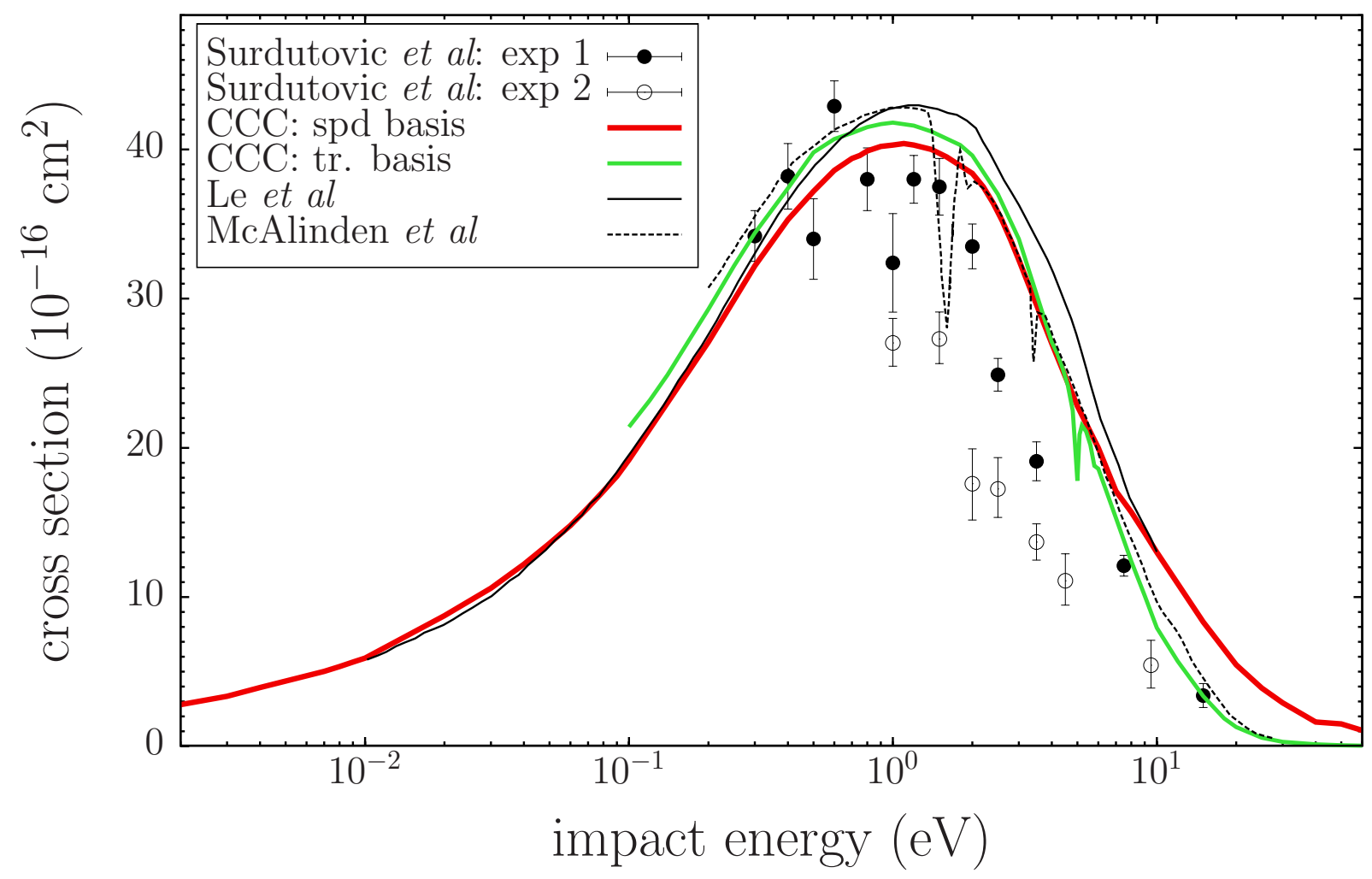

FIG. 6: Total positronium formation cross section for $\mathrm{e}^{+}-\mathrm{Li}$ along with the experimental points [35] and theoretical calculations [18, 23]. The CCC basis is the same as for Fig. 4. The truncated basis CCC calculation is an attempt to reproduce the states used by McAlinden et al. [18].

Direct scattering and positronium formation cross sections have been calculated for a broad range of energies of practical interest. Convergence in the calculated cross sections was demonstrated by increasing the basis sizes and orbital angular momentum of the included states for each of the centers. The obtained results are in good agreement with available theoretical data. Our calculations reveal a shallow Ramsauer-like minimum in the total and elastic cross sections near $0.0016 \mathrm{eV}$. We would appreciate further experimental investigation to see if the present discrepancy with experiment can be resolved.

\section{Acknowledgments}

The work was supported by the Australian Research Council. We are grateful for access to the Australian National Computing Infrastructure Facility and its Western Australian 
node Ive.

[1] C. M. Surko, G. F. Gribakin, and S. J. Buckman, J. Phys. B 38, 57 (2005).

[2] S. J. Gilbert, J. P. Sullivan, C. M. Surko, and S. J. Buckman, APS Meeting Abstracts pp. $5038-+(2001)$.

[3] A. Jones, P. Caradonna, C. Makochekanwa, D. Slaughter, D. Mueller, J. P. Sullivan, and S. J. Buckman, Journal of Physics: Conference Series 194, 012033 (2009), URL http://stacks . iop.org/1742-6596/194/i=1/a=012033.

[4] P. Caradonna, A. Jones, C. Makochekanwa, D. S. Slaughter, J. P. Sullivan, S. J. Buckman, I. Bray, and D. V. Fursa, Phys. Rev. A 80, 032710 (2009).

[5] A. Zecca, L. Chiari, A. Sarkar, M. A. P. Lima, M. H. F. Bettega, K. L. Nixon, and M. J. Brunger, Phys. Rev. A 78, 042707 (2008).

[6] A. Zecca, L. Chiari, A. Sarkar, K. L. Nixon, and M. J. Brunger, Phys. Rev. A 80, 032702 (2009).

[7] C. Makochekanwa, A. Bankovic, W. Tattersall, A. Jones, P. Caradonna, D. S. Slaughter, K. Nixon, M. J. Brunger, Z. Petrovic, J. P. Sullivan, et al., New Journal of Physics 11, 103036 (2009), URL http://stacks.iop.org/1367-2630/11/i=10/a=103036.

[8] P. A. Macri, J. E. Miraglia, J. Hanssen, O. A. Fojón, and R. D. Rivarola, Journal of Physics B: Atomic, Molecular and Optical Physics 37, L111 (2004), URL http://stacks.iop.org/ $0953-4075 / 37 / i=6 / a=L 01$.

[9] P. Macri and R. Barrachina, Nuclear Instruments and Methods in Physics Research Section B: Beam Interactions with Materials and Atoms 266, 393 (2008), ISSN 0168-583X, low Energy Positron and Positronium Physics - Proceedings of the XIV International Workshop on Low Energy Positron and Positronium Physics, URL http://www.sciencedirect.com/science/ article/B6TJN-4RC6R3T-9/\%2/e0d623f73357e739eed0832f5fb0e185.

[10] P. L. Bartlett, A. T. Stelbovics, T. N. Rescigno, and C. W. McCurdy, Phys. Rev. A 77, 032710 (2008).

[11] K. Bartschat, Phys. Rev. A 71, 032718 (2005).

[12] Y. Cheng and Y. Zhou, Phys. Rev. A 76, 012704 (2007).

[13] J. Mitroy, Australian Journal of Physics 46, 751 (1993). 
[14] G. Ryzhikh and J. Mitroy, Journal of Physics B: Atomic, Molecular and Optical Physics 30, 5545 (1997), URL http://stacks.iop.org/0953-4075/30/i=23/a=018.

[15] S. J. Ward, M. Horbatsch, R. P. McEachran, and A. D. Stauffer, Journal of Physics B: Atomic, Molecular and Optical Physics 22, 1845 (1989), URL http://stacks.iop.org/0953-4075/ $22 / i=11 / a=017$.

[16] K. P. Sarkar, M. Basu, and A. S. Ghosh, Journal of Physics B: Atomic, Molecular and Optical Physics 21, 1649 (1988), URL http://stacks.iop.org/0953-4075/21/i=9/a=022.

[17] P. Khan, S. Dutta, and A. S. Ghosh, Journal of Physics B: Atomic and Molecular Physics 20, 2927 (1987), URL http://stacks.iop.org/0022-3700/20/i=12/a=035.

[18] M. T. McAlinden, A. A. Kernoghan, and H. R. J. Walters, Journal of Physics B: Atomic, Molecular and Optical Physics 30, 1543 (1997), URL http://stacks.iop.org/0953-4075/ $30 / i=6 / a=021$.

[19] S. Guha and A. S. Ghosh, Phys. Rev. A 23, 743 (1981).

[20] M. Basu and A. S. Ghosh, Journal of Physics B: Atomic and Molecular Physics 19, 1249 (1986), URL http://stacks.iop.org/0022-3700/19/i=8/a=019.

[21] M. A. Abdel-Raouf, Journal of Physics B: Atomic, Molecular and Optical Physics 21, 2331 (1988), URL http://stacks.iop.org/0953-4075/21/i=12/a=019.

[22] R. N. Hewitt, C. J. Noble, and B. H. Bransden, Journal of Physics B: Atomic, Molecular and Optical Physics 25, 2683 (1992), URL http://stacks .iop.org/0953-4075/25/i=11/a=022.

[23] A.-T. Le, M. W. J. Bromley, and C. D. Lin, Phys. Rev. A 71, 032713 (2005).

[24] A. S. Kadyrov and I. Bray, Journal of Physics B: Atomic, Molecular and Optical Physics 33, L635 (2000), URL http://stacks.iop.org/0953-4075/33/i=18/a=101.

[25] R. Utamuratov, A. S. Kadyrov, D. V. Fursa, and I. Bray, Journal of Physics B: Atomic, Molecular and Optical Physics 43, 031001 (2010), URL http://stacks.iop.org/0953-4075/43/ $i=3 / a=031001$.

[26] R. Utamuratov, A. S. Kadyrov, D. V. Fursa, I. Bray, and A. T. Stelbovics, Journal of Physics B: Atomic, Molecular and Optical Physics 43, 125203 (2010), URL http://stacks .iop.org/ $0953-4075 / 43 / i=12 / a=125203$.

[27] A. S. Kadyrov and I. Bray, Phys. Rev. A 66, 012710 (2002).

[28] I. Bray, Phys. Rev. A 49, 1066 (1994).

[29] J. B. Furness and I. E. McCarthy, J. Phys. B 6, 2280 (1973). 
[30] B. J. Albright, K. Bartschat, and P. R. Flicek, Journal of Physics B: Atomic, Molecular and Optical Physics 26, 337 (1993), URL http://stacks.iop.org/0953-4075/26/i=3/a=008.

[31] J. Migdalek and W. E. Baylis, Phys. Rev. A 24, 649 (1981).

[32] I. Bray and A. T. Stelbovics, Phys. Rev. A 46, 6995 (1992).

[33] L. J. Radziemski, R. Engleman, and J. W. Brault, Phys. Rev. A 52, 4462 (1995).

[34] A. S. Kadyrov, I. Bray, and A. T. Stelbovics, Phys. Rev. Lett. 98, 263202 (2007).

[35] E. Surdutovich, J. M. Johnson, W. E. Kauppila, C. K. Kwan, and T. S. Stein, Phys. Rev. A 65, $032713(2002)$. 